\title{
Presentation of child sexual abuse cases to Queen Elizabeth Central Hospital following the establishment of an HIV post-exposure prophylaxis programme
}

\author{
Emily Chesshyre', Elizabeth M Molyneux ${ }^{2}$ \\ 1. Royal Devon and Exeter Hospital - Paediatrics, United Kingdom \\ 2. Department of Paediatrics, College of Medicine, Blantyre, Malawi \\ Correspondence: Dr. E Chesshyre Email: emilychesshyre@doctors.org. \\ uk
}

\section{Abstract}

\section{Aims}

To review child sexual abuse cases and their management presenting to Queen Elizabeth Central Hospital (QECH), Blantyre, since the introduction of an HIV post-exposure prophylaxis (PEP) programme.

\section{Methods}

Demographic and medical data was collected from all children presenting to Queen Elizabeth Central Hospital, Blantyre, Malawi between January 2005 and February 2007 with alleged child sexual abuse (CSA).

\section{Results}

Between January 2005 and February 2007, 217 children presented with alleged CSA. This is an average of 3 more per month since the previous year. The results of the physical examination in $60 \%(130 / 217)$ of the cases showed signs of trauma. $63 \%(137 / 217)$ of the cases presented within 72 hours of defilement. Overall in 42\% (92/217) of children a one month course of HIV PEP was indicated and given. In 58\% (125/217) HIV PEP was not indicated in view of normal examination, presentation too late $(>72 \mathrm{hrs}$ after abuse), multiple abuse episodes in the last 6 months, HIV test positive or HIV test refused. In 66\% (144/217) of assessed children antibiotic treatment was given for the prevention and/ or treatment of sexually transmitted infections (STIs).

\section{Conclusions}

The introduction of an HIV PEP programme for victims of CSA has lead to increased numbers presenting and being treated. In conclusion it is likely that a significant number of children have been prevented from acquiring HIV and other STIs following CSA. The key area where our service needs to be improved is in establishing documented follow up of all cases to monitor medication compliance, side effects and rates of HIV seroconversion following CSA.

\section{Introduction}

In January 2004 a programme to provide HIV post-exposure prophylaxis (PEP) to eligible children suffering from child sexual abuse (CSA) was established in Queen Elizabeth Central Hospital, Blantyre, Malawi ${ }^{1}$. This was in view of the high HIV seroprevalance in Malawi, the risk of children contracting HIV following CSA and the availability of antiretroviral therapy (ARVs).

The presumed mechanism of action of HIV PEP is that shortly after exposure to HIV the viral load is small enough to be controlled by the body's immune system, and ARVs at this time may end viral replication ${ }^{2}$. For this reason HIV PEP is recommended in many circumstances, including risk reduction of mother to child transmission of $\mathrm{HIV}^{3}$ and occupational exposure to $\mathrm{HIV}^{4}$. United Kingdom and American guidelines recommend HIV PEP for children after sexual exposure depending on the risk of seroconversion $5,6,7,8$. HIV PEP is recommended as part of post-rape care in high prevalence areas such as South Africa?

Risk of HIV transmission is calculated as the likelihood that the source is HIV infected multiplied by the type of exposure ${ }^{5}$. With the high HIV seroprevalance in Malawi, estimated prevalence in adults (15-49 yrs) in 2007 was $11.9 \%$, there is a substantial risk that a perpetrator is HIV infected. The risk of exposure is especially high in a sexually abused premenarchial girl when there has been an associated trauma. This is due to increased HIV transmission in the presence of cervical ectopy and an immature thin premenarchial vaginal epithelium $^{8,11}$. A service which provides HIV PEP was deemed essential in the central teaching hospital in Blantyre, Malawi and was set up in January 2004. During the first year, 64 children presented with alleged child sexual abuse ${ }^{1}$. Here we present the numbers presenting between January 2005 and February 2007.

\section{Methods}

Queen Elizabeth Central Hospital (QECH) is a tertiary level referral hospital in Blantyre, Southern Malawi. In January 2002 a standardised clinical assessment ${ }^{12}$ form was introduced to assess children presenting as victims of CSA. Demographic data is collected, details and timings of alleged assault are recorded. A full medical history is taken and systemic and anogenital examination performed and recorded by a specialist paediatric doctor (registrar or consultant) or specialist paediatric clinical officer, with nurse presence, in a designated private room for the purpose, containing necessary investigations and medication in a locked cupboard.

From January 2004 a written protocol was introduced to determine eligibility for HIV $\mathrm{PEP}^{13}$. Children with a history of alleged sexual abuse are considered eligible for PEP if they are seen less than 72 hours after the abuse, have not been previously assaulted in the last 6 months, there are signs of trauma (vaginal or anal) on medical examination (for specific signs see reference 24 ), they are HIV sero-negative on testing on the day of presentation (test undertaken after pre-counselling), and the child and guardian agree to comply with treatment.

If the child is eligible for PEP they were given a 30 day course of zidovudine and lamivudine, dosed according to body weight. All children are offered follow up in the general paediatric clinic or paediatric $A \& E$ one month from presentation. If the child is found to be HIV positive on testing they are referred to the paediatric antiretroviral clinic. If the child is not eligible for PEP they are offered an HIV test if indicated and a repeat test in 3 months.

In addition to HIV PEP, where there is a history of alleged CSA children are offered antibiotics to help prevent other 


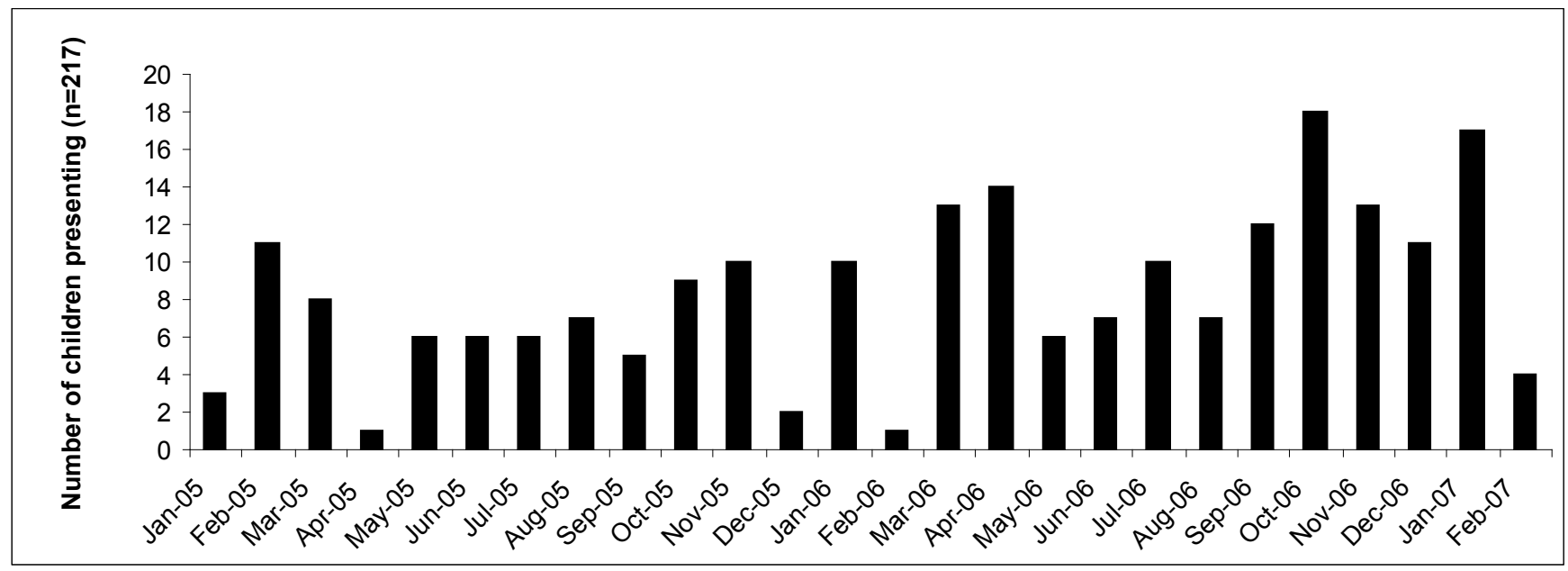

sexually transmitted infections (STIs). If there is evidence of trauma, tetanus toxoid and need for surgical intervention is considered. Pregnancy testing is offered to children postmenarche. If it is felt that the child is vulnerable to repeated abuse at home they are admitted and social services are asked to investigate the home circumstances.

Data was collected retrospectively in March 2007 from the completed standardised clinical assessment forms. Clinical assessment forms are stored in a locked office only accessible to immediate service providers. Data collected from forms was anonymised.

\section{Results}

Between January 2005 and February 2007, 217 children presented with alleged CSA. This is an average of 8.3 (range 1-18) children/ month. The overall trend of number of cases is increasing (see figure 1), with an average of $6.5 /$ month presenting in the 13 months from January 2005 to January 2006 an average of 10.2 /month presenting from February 2006 to February 2007 ( $p=0.035$ ) (Mann-Whitney test comparing monthly attendance between two time frames). In 200464 children (average 5.3/month) presented with alleged CSA ${ }^{1}$. There have been on average an extra 3 cases/ month since 2004 .

Fifty two percent $(113 / 217)$ of children were in the age group 5-10 years old, 29\% (62/217) were under 5 years old and $19 \%(42 / 217)$ were $11-16$ years old. The median age was 7 years old, ranging from 14 months to 16 years.

Almost all $(95 \%, 207 / 217)$ of the victims were girls and $5 \%$ $(10 / 217)$ male. Of the female children 96\% (198/207) had not yet started menses.

A single alleged defilement episode was reported in $65 \%$ $(142 / 217)$ of cases. $25 \%(54 / 217)$ reported multiple episodes. In $10 \%(21 / 217)$ of cases the child did not report defilement, but it was suspected on other grounds.

Time from the last alleged incident was less than 72 hours in $63 \%(137 / 217)$ of cases. In $29 \%(62 / 217)$ of cases it was more than 72 hours. Timing was not given in $8 \%(18 / 217)$ of cases.

The alleged perpetrator was known to the child in $65 \%$ $(141 / 217)$ cases, and unknown in 35\% (76/217) cases. Where the alleged perpetrator was known, in $16 \%$ cases $(22 / 141)$ they were a family member.

Vaginal rape was alleged in $81 \%(175 / 217)$ of cases, anal rape in $6 \%(14 / 217)$ of cases, and other types of sexual assault, or details were not provided by the child in $13 \%(28 / 217)$ of cases.

The results of the physical examination in 60\% (130/217) of cases showed signs of trauma. In the remaining $40 \%$ $(87 / 217)$ of cases the examination was normal.

In no cases was the HIV status of the alleged perpetrator known.

Of those offered an HIV spot test $92.8 \%(142 / 153)$ were non-reactive, $4.6 \%(7 / 153)$ were reactive and $2.6 \%(4 / 153)$ refused testing. Those who were found to be HIV positive were referred to the paediatric ARV clinic.

Overall in 42\% (92/217) of children HIV PEP was indicated and given, in 58\% (125/217) HIV PEP was not indicated as examination was normal, presentation too late ( $>72 \mathrm{hrs}$ ), multiple episodes of abuse had occurred over a period of time, the child's HIV test was positive or an HIV test was refused.

In $66 \%(144 / 217)$ of assessed children antibiotic treatment was given for the prevention/ treatment of STIs.

\section{Discussion}

\section{Changes since implementation of HIV PEP service for CSA in QECH}

We can see that since the establishment of this service the number of children brought for assessment and management have increased. This could be because the incidence of CSA is rising, or because more children are attending now that HIV PEP is available. CSA is reported to be increasing in South Africa ${ }^{14}$, but decreasing in $\mathrm{USA}^{15}$. In other areas in Africa when similar services were established, unexpectedly high numbers presented ${ }^{16,17}$. It is likely that we are seeing only a small proportion of the total numbers of cases.

It is important that we rise to the challenge to provide more comprehensive care to more victims of CSA. This includes not only medical interventions of HIV PEP, and prevention or treatment of other STIs, but access to psychology services 
to offer follow up, counselling and support.

Our audit found a high number of children with positive physical examination findings. This differs greatly from countries such as the USA, where positive physical examination findings are only found in $4 \%$ of cases ${ }^{18}$. Possible reasons for this are that in Malawi families may not present unless there are physical signs of injury. The QECH is a tertiary referral hospital and may only be referred the most severe cases. Or it may be because the majority of referrals come from the police who may only refer severe cases.

\section{Training implications}

With the increase in cases of child sexual abuse there is the need for more staff to be trained in the medical assessment and examination of these children. In our hospital child sexual abuse assessments are carried out by paediatric specialist doctors or clinical officers. To our knowledge, QECH is the only hospital in Malawi that provides this service in a formalised manner. However CSA happens in all communities. The establishment of training courses for paediatric medical staff in district hospitals would enable more children to receive timely HIV PEP in eligible circumstances. The importance of proper and correct physical examination and documentation cannot be overemphasised as this has implications not only on the child's treatment, but also legal implications if a perpetrator is brought before the court. Both training and experience are necessary to be able to carry out such examinations. Both variants of normal genitalia, and subtle signs of abuse, must be interpreted correctly. Examination must be done in a standardised manner by a trained clinician. Findings need to be accurately documented in a clear format that is understandable to clinicians, police and public. A national standardised clinical assessment form such as the one used in $\mathrm{QECH}^{12}$ would be helpful towards developing standardised assessments.

\section{Importance of one stop service}

A key learning point for our department in setting up this service was the importance of offering assessment, examination, pre HIV test counselling, HIV testing and HIV PEP administration, if indicated, at the same point of care with 24 hour accessibility. This enables the maximum number of children to benefit from HIV PEP where indicated, rather than some missing out if unable to complete the process when sent to different departments of the hospital for some of the treatments or tests. This has caused a high drop out rate in HIV PEP programmes elsewhere ${ }^{17}$. Having a one stop service also minimises time delay in receiving HIV PEP, in a situation that is time critical. Furthermore if indicated, the full 30 day course of treatment is provided on the day of assessment. In KwaZulu-Natal only the first week of treatment was dispensed, requiring patients to return at one week and regularly after that to be able to complete the full treatment course ${ }^{16}$. In the recent Refentse intervention study in rural South Africa provision of the full course of treatment on the day of assessment is strongly recommended to enhance adherance ${ }^{25}$.

\section{Problems with our service: time of presentation}

HIV PEP is most effective when taken as soon as possible after exposure, preferably within the hour ${ }^{6,19}$. The longer time from exposure, the less likely PEP is to be effective, so that after 72 hours post exposure it is rarely indicated ${ }^{7,20}$.

In our sample $29 \%(62 / 217)$ of children presented more than 72 hours after the event, thus excluding them being eligible for HIV PEP. This result is similar to findings in South Africa ${ }^{16}$.

In Malawi there are inevitable delays in presentation. Firstly it is likely that the many of the victims do not present at all, or have a delayed presentation in view of stigma, lack of family support, and transport difficulties ${ }^{1}$. Of those that presented the vast majority were referred from the police, as the legal notification is deemed more important than medical assessment. By seeing the police first this creates a delay in receiving HIV PEP. However it does create a certain pathway of referral.

This could be improved by education of the public of the benefit of coming for medical assessment following CSA, and of the importance of coming as soon as possible after the event.

\section{Problems with our service: follow up}

A key problem with our service is providing long term follow up. All children who received PEP were given follow up appointments for one month time to monitor for side effects, compliance and repeat HIV testing. Follow up was offered either at the paediatric A\&E, or at paediatric general outpatients clinic. As the children were seen by different clinicians and the follow up consultations recorded in the child's health passport, we were unable to collect data on numbers that returned for follow up. This means we have no data available on compliance with the 30 day course of HIV PEP, or the results of follow up HIV tests. Therefore we do not know the efficacy of our service. In previous studies in Africa adherence rates to HIV PEP in CSA has been poor in Zambia ${ }^{21}$, Kenya ${ }^{17}$ and Malawi ${ }^{1}$. Mean adherence rates in these studies was $55 \%$ (range: $44.8-64.7 \%)^{16}$. In KwaZuluNatal, South Africa adherence rates in CSA survivors were $35.4 \%$ who returned for the full 28 day course, and only $3.5 \%$ who returned for both 3 and 6 month follow-up ${ }^{16}$. This could be due to factors such as lack of understanding of the need for follow up, difficulties in transportation, adverse drug reactions, poor social support and traumatic associations of the assault ${ }^{1,16,17}$. In addition to reduced efficacy, poor adherence to medication may theoretically lead to acquisition of a drug resistant virus, should the individual become HIV infected ${ }^{5}$.

Without follow up, side effects of medication cannot be monitored. This is necessary to know to help determine the risk benefit ratio of providing treatment. In Kenya there were few reported side effects in children, with no haematological or hepatotoxicity on the same PEP drug regime as used in Malawi ${ }^{17}$.

Lack of follow up for those not eligible for HIV PEP is just as important to enable repeat HIV testing. In KwaZululNatal, of those who returned for follow up who had not been eligible for PEP due to delayed presentation $>72$ hours, $25 \%(2 / 8)$ became HIV positive, suggesting that this is a high risk group ${ }^{16}$. Undetected seroconversion in both PEP treated and non PEP treated groups poses a significant risk both to the individual and community ${ }^{16}$. Thus to provide an effective service, recordable follow up is essential. Our service needs 
to be developed to provide documented follow up to be able to monitor the efficacy of treatment. In addition children and guardians need to be educated on the importance of compliance and attendance of follow up appointments.

\section{Should we provide HIV PEP to more CSA victims?}

It has been argued that the potential benefit of offering HIV PEP to more victims of CSA outweighs the risks. In particular it is felt by some that HIV PEP should still be given if there have been multiple CSA episodes in the last 6 months, and the child is HIV negative on testing ${ }^{22}$. It is not offered in our service in these circumstances in view of the possibility of having already acquired HIV, but being in the window period of seroconversion. However others argue that this is not necessarily the case and in some of these cases providing HIV PEP may prevent the acquisition of HIV from the most recent assault ${ }^{22}$. Guardians would need to be fully counselled on the possibility of becoming seropositive if they are in fact in the window period of seroconversion. In our group $25 \%$ (54/217) had suffered multiple defilement episodes, thus excluding them from treatment with HIV PEP.

In addition, in our service HIV PEP is withheld if there is a normal physical examination. This is because although it is known that sexual penetration can occur without causing physical signs on examination, if there are no signs of trauma the risk of HIV acquisition is much lower. However others argue the need to consider HIV PEP in these cases in countries with high HIV prevalence ${ }^{22}$. Ellis et $\mathrm{al}^{1}$ have argued that in this situation the risks of treatment outweigh the benefits. However in studies from Kenya ${ }^{17}$ and South Africa ${ }^{16,25}$ both with a similar adult HIV prevalence to Malawi ${ }^{10}$, children with a normal physical examination were not excluded. The American Academy of Pediatrics advise considering PEP in cases without trauma ${ }^{8}$, a country with a much lower incidence of HIV prevalence ${ }^{10}$. Thus providing HIV PEP to otherwise eligible children with normal examination findings should be re-considered in our setting. Some guidelines recommend the consideration of HIV PEP after 72 hours in high risk circumstances $^{5}$. However animal models of PEP have shown no benefit of PEP if begun after 72 hours of exposure ${ }^{23}$.

It is firmly established that if the family refuse testing they should not be offered HIV PEP due to the risk that they may already be HIV positive and HIV PEP could lead to the development of drug resistance in that individual ${ }^{5}$.

In summary the benefit needs to outweigh the risks of treatment, which are side effects of medication, potential development of drug resistance and cost. The decision to start HIV PEP needs to occur on a patient to patient basis.

\section{Recommendations}

\section{Provision of HIV PEP provides an opportunity to improve services for victims of CSA}

Provision of HIV PEP for CSA not only prevents some children from acquiring HIV from CSA, but attracts more children and guardians to the service, as well as interprofessional referral to the service. Thus they can be offered further medical and non-medical services in addition to HIV PEP. Other medical treatments include antibiotics to prevent and/or treat other STIs, repair of gross injuries and tetanus toxoid if indicated. Non-medical services include the provision of social and psychological services. Currently there are no psychological services available to victims of CSA in QECH but this is an area which would greatly benefit children and could be developed within the context of our service. The best model for the victim would be to have a one stop multidisciplinary approach including police, forensic, social, psychological and legal support services. One successful model shown in the Refentse intervention study, in a rural South African hospital, is a nurse driven model of comprehensive post rape care ${ }^{25}$.

As stated previously the next essential development of our service is adequate recordable follow up. This would best be done at the same point of contact of the initial presenting visit. Standardised forms should be completed to enable data collection of patients followed up.

The next stage would be to expand the service beyond the tertiary hospital to district hospitals. This could only be done after a programme of training of a nominated paediatric clinician from each district hospitals, and with the use of a national standardised clinical assessment form. If the training was fully comprehensive this would allow the clinician to carry out assessment of CSA cases in their district hospital with HIV PEP provision. This would enable a greater coverage of HIV PEP to more eligible victims of CSA, in a more timely manner.

\section{References}

1. Ellis JC, Ahmad S, Molyneux EM. Introduction of HIV postexposure prophylaxis for sexually abused children in Malawi. Arch Dis Child 2005;90:1297-1299.

2. Merchant RC, Keshavarz R. Human Immunodeficiency Virus post exposure prophylaxis for adolescents and children. Pediatrics 2001;108(2):e38.

3. Volmink J, Siegfried NL, van der Merwe L, Brocklehurst P. Antiretrovirals for reducing the risk of mother-to-child transmission of HIV infection. Cochrane Database Syst Rev 2007:1:CD003510.

4. Young TN, Arens EJ, Kennedy GE, Laurie JW, Rutherford G. Antiretroviral post-exposure prophylaxis (PEP) for occupational HIV exposure. Cochrane Database Syst Rev 2007;1:CD002835

5. Fisher M, Benn P, Evans B, Pozniak A, Hawkins D et al. UK Guideline for use of PEP for HIV following sexual exposure. International Journal of STD and AIDS 2006;17:81-92.

6. Tudor-Williams G. Post-exposure prophylaxis Guidelines for children exposed to blood borne viruses. 2007. www.CHIVA.org.uk/ protocols/pep.html.

7. Smith DK, Grohskopf LA, Black RJ, Auerback JD, Greenberg AE et al. Antiretroviral postexposure prophylaxis after sexual, injectiondrug use, or other non-occupational exposure to HIV in the United States. Centers for Disease Control and Prevention. MMWR Recommendations and Reports 2005;54(RR02):1-20. http://www. cdc.gov/mmwr/preview/mmwrhtml/rr5402a1.htm

8. Havens PL et al. Postexposure prophylaxis in Children and Adolescents for Nonoccupational exposure to HIV. Pediatrics 2003;111:1475-1489.

9. Kim JC, Martin LJ, Denny L. Rape and HIV post-exposure prophylaxis: addressing the dual epidemics in South Africa. Reprod Health Matters 2003;11(22):101-112.

10. Unicef. Statistics: Malawi. 2005. http://www.unicef.org/ infobycountry/malawi_statistics.html.

11. Gutman LT, Herman-Giddens ME, McKinney RE Jr. Pediatric acquired immunodeficiency syndrome. Barriers to recognising the role of child sexual abuse. Am J Dis Child 1993;147:775-780.

12. Robson J. Medical Assessment for Defilement. Appendix 1 for Ellis JC, Ahmad S, Molyneux EM. Introduction of HIV post-exposure 
prophylaxis for sexually abused children in Malawi. Arch Dis Child 2005;90:1297-1299. See www.archdischild.com/supplemental.

13. Ellis JC. Guidelines for Post- Exposure Prophylaxis after Sexual Abuse. Appendix 2 for Ellis JC, Ahmad S, Molyneux EM. Introduction of HIV post-exposure prophylaxis for sexually abused children in Malawi. Arch Dis Child 2005;90:1297-1299. See www.archdischild. $\mathrm{com} /$ supplemental.

14. Collings SJ, Wiles WA. Child rape in KwaZulu-Natal, South Africa: An analysis of substantiated cases. Acta Criminologica 2004;17(3):4854.

15. Jones LM, Finkelhor D. Putting together evidence on declining trends in sexual abuse: a complex puzzle. Child Abuse Negl 2003;27(2):133135.

16. Collings SI, Bugwandeen SR, Wiles WA. HIV post-exposure prophylaxis for child rape survivors in Kwazulu-Natal, South Africa: Who qualifies and who complies? Child Abuse Negl 2008;32(4):47783.

17. Speight CG, Klufio A, Kilonzo SN, Mbugua C, Kuria E, Bunn JE, Taegtmeyer M. Piloting post-exposure prophylaxis in Kenya raises specific concerns for the management of childhood rape. Transactions of the Royal Society of Tropical Medicine and Hygiene 2006;100:14-18.

18. Johnson CF. Child sexual Abuse. Lancet 2004;364:462-70.
19. Boyles T, Freedman A. Post- exposure prophylaxis. Medicine 2005;33(6):32-33.

20. Prins JM. HIV post-exposure prophylaxis after occupational and nonoccupational exposure. Gynaecology, Obstetrics, and Reproductive Medicine in Daily Practice 2005;1279:105-108.

21. Haworth A. Problems in establishing post-exposure prophylaxis for HIV in sexually abused children in Zambia. In Paper presented at the 16th ISPCAN International Congress on Child Abuse and Neglect 2006.

22. Muula AS. HIV post-exposure prophylaxis for sexually abused children in Malawi. Arch Dis Child eLetters for Ellis et al. 2005;90(12):1297-1299.

23. Martin LN, Murphey-Corb M, Soike KF, Davison-Fairburn B, Baskin GB. Effects of initiation of 3'-azido, 3'-deoxythymidine (zidovudine) treatment at different times after infection of rhesus monkeys with simian immunodeficiency virus. J Infec Dis 1993;168:825-835.

24. Adams JA, Kaplan RA, Starling SP, Mehta MH, Finkel MA, Botash AS, Kellogg ND, Shapiro RA. Guidelines for medical care of children who may have been sexually abused. J Pediatr Adolesc Gynaecol 2007;20:163-172.

25. Kim J, Askew I, Muvhango L, Dwane N, Abramsky T, Jan S, Ntlemo E, Chege J, Watts C. Comprehensive care and HIV prophylaxis after sexual assault in rural South Africa: the Refentse intervention study. BMJ 2009;338:b515.

\section{Ipas trains the media on unsafe abortions}

Ipas an international organization that promotes reproductive health for women organized a three-day workshop from 30th March to 1st April on how the media can effectively report on safe abortion. According to statistics presented by the vice president for Ipas in Africa, Ambassador Dr. Brookman Amissah, abortions accounts for 1 in 8 maternal deaths globally and that $13 \%$ of these are from unsafe abortions. She cited that lack of access to effective contraception, stigma and silence are a major cause of unsafe abortions particularly in Africa. "There is ignorance among policy makers, legislators, health professionals and amongst women in the communities themselves, that's why we end up loosing lives which could have been saved".

Making his presentation at the workshop the coordinator for Ipas in Malawi who is also a legal practitioner Mr. Godfrey Kangaude, mentioned that when reporting about abortion law, journalists should not forget that there is also a right to life. "The media has a responsibility to help the public to reflect to what extent abortion should be legal in the country and should help the public discuss what the preservation of the mother's life should mean for Malawians", he said.
Abortion is illegal in Malawi and according to the penal code any person who provides abortion is guilty of a felony and liable to prison for fourteen years. Women who procure abortions are guilty and liable to a seven year sentence. While reading the legislation, Kangaude revealed that it is safe to terminate a pregnancy if it poses a risk to the life of the woman and that person would not be criminally liable.

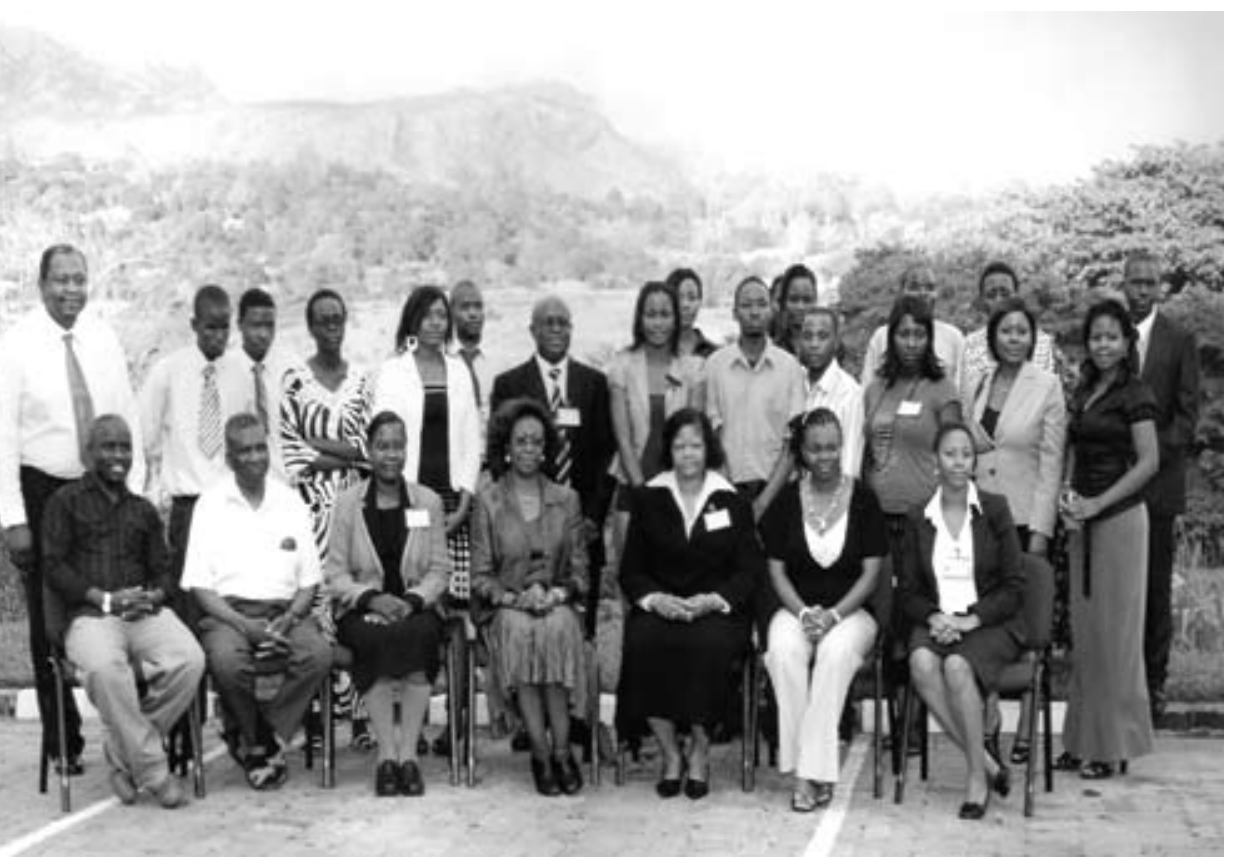

Ipas and brought together officials from the Ministry of Health, legal practitioners, human rights activists and journalists from different media houses. .

Ipas is an international organization that works around the world to increase women's ability to exercise their sexual and reproductive rights, and to reduce abortion-related deaths and injuries.

The objectives were to educate participant s on issues of abortion, raise awareness on the commitments made by governments at regional and international levels to reduce unsafe abortions, and to report on abortion issues from a human rights perspective.

The workshop was organized by $\mathrm{O}$ media in conjunction with conjunction with 